\title{
A Note on Some Equivalences of Operators and Topology of Invariant Subspaces
}

\section{Bernard Mutuku Nzimbi}

School of Mathematics, College of Biological and Physical Sciences, University of Nairobi, Nairobi, Kenya

\section{Email address:}

nzimbi@uonbi.ac.ke

\section{To cite this article:}

Bernard Mutuku Nzimbi. A Note on Some Equivalences of Operators and Topology of Invariant Subspaces. Mathematics and Computer Science. Vol. 3, No. 5, 2018, pp. 102-112. doi: 10.11648/j.mcs.20180305.12

Received: January 8, 2018; Accepted: February 7, 2018; Published: December 28, 2018

\begin{abstract}
In this paper we investigate the invariant and hyperinvariant subspace lattices of some operators. We give a lattice-theoretic description of the lattice of hyperinvariant subspaces of an operator in terms of its lattice of invariant subspaces. We also study the structure of these lattices for operators in certain equivalence classes of some equivalence relations.
\end{abstract}

Keywords: Invariant Subspace, Reducing Subspace, Hyperinvariant, Hyper-Reducing, Commutant, Bicommutant, Reducible, Irreducible Operator

\section{Introduction}

In this paper $H$ will denote a complex separable Hilbert space and $B(H)$ will denote the Banach algebra of bounded linear operators. If $T \in B(H)$, then $T^{*}$ denotes the adjoint of $T$, while $\operatorname{Ker}(T), \operatorname{Ran}(T), \bar{M}$ and $M^{\perp}$ stands for the kernel of $T$, range of $T$, closure of $M$ and orthogonal complement of a closed subspace $M$ of $H$, respectively. Recall that an operator $T \in B(H)$ is normal if $T^{*} T=T T^{*}$, unitary if $T^{*} T=T T^{*}=I$, a projection(or idempotent) if $T^{2}=T$, an orthogonal projection if $T^{2}=T$ and $T^{*}=T$. An operator $T \in B(H)$ is said to be scalar if it is a scalar multiple of the identity operator. That is, if $T=\alpha I$, where $\alpha \in \mathbb{C}$ and $I$ is the identity operator on $H$. An operator $T \in B(H)$ is compact if for each bounded set $M \subseteq H$, then the closure of the image $\overline{T(M)}$ is compact. This is equivalent to saying that $\overline{T(B(0,1))}$ is compact, where $B(0,1)=\{x \in H:\|x\|<1\}$. An operator $T \in B(H)$ is polynomially compact if there exists a non-zero polynomial $p$ such that $p(T)$ is compact.

Two operators $A \in B(H)$ and $B \in B(K)$ are said to be similar if there exists an invertible operator $N \in B(H, K)$ such that $N A=B N$ or equivalently $A=N^{-1} B N$, and are unitarily equivalent if there exists a unitary operator $U \in B_{+}(H, K)$ (Banach algebra of all invertible operators in $B(H)$ ) such that $U A=B U$ (i.e. $A=U^{*} B U$ equivalently, $\left.A=U^{-1} B U\right)$. An operator $X \in B(H, K)$ is a quasiaffinity or a quasi-invertible if it is injective and has dense range. Two operators $A \in B(H)$ and $B \in B(K)$ are said to be quasiaffine transforms of each other if there exists a quasiaffinity $X \in B(H, K)$ such that $X A=B X$.

Two operators $A \in B(H)$ and $B \in B(K)$ are quasisimilar if there exist quasiaffinities $X \in B(H, K)$ and $Y \in B(K, H)$ such that $X A=B X$ and $A Y=Y B$. (see [11], [14]).

For any operators $A, B \in B(H)$, we define $[A, B]=A B-B A$.

The commutant of $T \in B(H)$ denoted by $\{T\}^{\prime}$ is the set of all operators that commute with $T$. That is, $\{T\}^{\prime}=\{S \in B(H): S T=T S\}$. (see [4]).

The bicommutant or double commutant of $T \in B(H)$ denoted by $\{T\}^{\prime \prime}$ is defined by

$$
\{T\}^{\prime \prime}=\left\{A \in B(H): A S=S A, S \in\{T\}^{\prime}\right\}
$$


It is clear that

$$
\{T\}^{\prime \prime}=\{p(T): T \in B(H)\}=\bigcap_{S \in\{T\}^{\prime}}\{S\}^{\prime} .
$$

A subspace $M \subseteq H$ is said to be invariant under $T \in B(H)$ if $T M \subseteq M$. In this case, we say that the subspace $M$ is $T$-invariant.

A subspace $M \subseteq H$ is said to be a reducing subspace of $T \in B(H)$ if it is invariant under both $T$ and $T^{*}$ (equivalently, if both $M$ and $M^{\perp}$ are invariant under $T$ ). For more details about invariant subspaces and the Invariant Subspace Problem (see [11], [14] and [17]).

A subspace $M \subseteq H$ is said to be a hyperinvariant subspace for $T \in B(H)$ if $S M \subseteq M$ for each $S \in\{T\}^{\prime}$. That is, it is invariant under every operator commuting with $T$.

A lattice is a partially ordered set in which every pair of elements has a least upper bound and a greatest lower bound.

By a subspace lattice on a Hilbert space $H$ we mean a family of subspaces of $H$ which is closed under the formation of arbitrary intersections and arbitrary linear spans and which contains the zero subspace $\{0\}$ and $H$.

The subspace lattice of all invariant, reducing and hyperinvariant subspaces of $T$ is denoted by $\operatorname{Lat}(T)$, $\operatorname{Re} d(T)$ and HyperLat $(T)$, respectively. Note that these lattices are complete, in the sense that intersections and closed linear spans of subspaces are also in these lattices. Since $T$ commutes with itself, we have that

Hyperlat $(T) \subseteq \operatorname{Lat}(T)$. (see [4] and [12]).

It is also true that

$$
\operatorname{Re} d(T) \subseteq \operatorname{Lat}(T) .
$$

We sometimes call the lattice of hyperinvariant subspaces an hyperlattice.

Let $A, B \in B(H)$. We say that an operator $T \in B(H)$ intertwines the pair $(A, B)$ if $T A=B T$. If $T$ intertwines both $(A, B)$ and $(B, A)$, then we say that $T$ doubly intertwines $A$ and $B$.

A quasiaffinity $X$ is said to have the hereditary property with respect to an operator $T \in B(H)$ if $X \in\{T\}^{\prime}$ and $\overline{X(M)}=M$ for every $M \in \operatorname{Hyperlat}(T)$. If $T_{1}$ and $T_{2}$ are quasisimilar and there exists an implementing pair $(X, Y)$ of quasiaffinities such that $X Y$ has the hereditary property with respect to $T_{1}$ and $Y X$ has the hereditary property with respect to $T_{2}$, then we say that $T_{1}$ is hyper-quasisimilar to $T_{2}$. This is denoted by $T_{1} \approx T_{2}$. The notion of hyperquasisimilarity was introduced by C. Foias etal[2].

We note that hyper-quasisimilarity is strictly stronger than quasisimilarity (see [2], Proposition 2.7). In fact the following inclusion of operator equivalences is true.

$$
\text { Similar } \subset \text { Hyper }- \text { quasisimilar } \subset \text { Quasisimilar } .
$$

We call $a \subseteq B(H)$ a subalgebra of $B(H)$ if $a$ is closed under scalar multiplication, addition and composition. If $a$ is also closed under taking adjoint, we call it a *-subalgebra of $B(H)$. If the identity operator $I$ belongs to the subalgebra $a$, we say that $a$ is a unital subalgebra of $B(H)$.

We denote by $W^{*}(T)$ the (unital) weakly closed von Neumann algebra generated by $T$. We will use this subalgebra to investigate the structures of invariant and hyperinvariant subspace lattices for some operators.

Two lattices $L_{1}$ and $L_{2}$ are said to be isomorphic (denoted by $L_{1} \equiv L_{2}$ ) if there exists an isomorphism (bijective map) $\varphi: L_{1} \rightarrow L_{2}$, and if $l_{1} \leq l_{2}$ if and only if $\varphi\left(l_{1}\right) \leq \varphi\left(l_{2}\right)$ for $l_{1}, l_{2} \in L_{1}$. (see [12]).

Let $T \in B(H)$. If $M$ reduces every operator in the commutant of $T$, then we call $M$ hyper-reducing subspace for $T$. We denote by Hyper $\operatorname{Re} d(T)$ the collection of all subspaces hyper-reducing for $T \in B(H)$. Clearly

$$
\text { Hyper } \operatorname{Re} d(T) \subseteq \operatorname{Lat}(T) \text {. }
$$

The concept of hyper-reducibility of a subspace of a Hilbert space was introduced by Moore[15]. We will prove in Section 5 that

$$
\text { Hyper } \operatorname{Re} d(T)=\operatorname{Re} d\left(\{T\}^{\prime}\right)=\operatorname{Lat}\left(\{T\}^{\prime}\right) \bigcap \operatorname{Lat}\left(\left\{T^{*}\right\}\right) .
$$

An operator $T \in B(H)$ is said to be reducible if it has a nontrivial reducing subspace (equivalently, if it has a proper nonzero direct summand-that is, if there exists a subspace $M$ of $H$ such that $M$ and $M^{\perp}$ are nonzero and $T-$ invariant)(see [14]). This is equivalent to saying that if $M$ is nontrivial and invariant under $T$ and $T^{*}$. A subspace that is not reducible is said to be irreducible. This means that an operator is irreducible if it has no reducing subspace other than $\{0\}$ and $H$. It has been shown in [8] that an operator $T \in B(H)$ is reducible if and only if there exists a non-scalar operator $L$ such that $L T=T L$ and $T^{*} L=L T^{*}$. That is if and only if there exists a non-scalar operator $L \in\{T\}^{\prime} \cap\left\{T^{*}\right\}^{\prime}$. Equivalently, $T$ is reducible if and only if both $T$ and $T^{*}$ lie in $\{L\}$ for some non-scalar operator $L$.

We denote by $\left\langle\left(\begin{array}{l}x \\ y\end{array}\right)\right\rangle$ the span of vector $\left(\begin{array}{l}x \\ y\end{array}\right)$.

\section{Main Results}

Recall that the commutant $\{a\}^{\prime}$ of a *-subalgebra $a \subseteq B(H)$ is the set 


$$
\{a\}^{\prime}=\{B \in B(H): B A=A B, \forall A \in a\} .
$$

Clearly $\{a\}^{\prime}$ is weakly closed. Since the weak operator topology is weaker than the strong operator topology, it is also clear that $\{a\}$ is always strongly closed.

Theorem 2.1 If $T \in B(H)$ then the following statements hold.

(i). $\{T\}^{\prime}$ is a subalgebra of $B(H)$.

(ii). $\{T\}$ is a commutative subalgebra of $B(H)$.

(iii). $\{T\}^{\prime \prime}=\left\{\{T\}^{\prime}\right\}^{\prime}$.

We need the following result which verifies a useful property of unital self-adjoint subalgebras of $B(H)$.

Lemma 2.2 Suppose $a$ is a self-adjoint subalgebra of $B(H)$ and let $M$ be a closed subspace of $H$. Then following statements are equivalent.

(i). $a(M) \subseteq M$.

(ii). $a\left(M^{\perp}\right) \subseteq M^{\perp}$.

(iii). $\left[a, P_{M}\right]=0$, where $P_{M}$ denotes the orthogonal projection of $H$ onto $M$.

Proof. (i) $\Rightarrow$ (ii): Suppose $a(M) \subseteq M$. That is $A y \in M$ for all $A \in a$ and $y \in M$. Let $x \in M^{\perp}$. Then

$$
\langle y, A x\rangle=\left\langle A^{*} y, x\right\rangle=0 .
$$

Since $A^{*} \in a$ we have that $A x \in M^{\perp}$, so $a\left(M^{\perp}\right) \subseteq M^{\perp}$.

(ii) $\Rightarrow$ (i): $a\left(M^{\perp}\right) \subseteq M^{\perp}$ implies that $a(M) \subseteq M$ follows from the fact that $M=M^{\perp}$.

(i) $\Rightarrow$ (iii): Suppose that $a(M) \subseteq M$ and let $A \in a$ and $x \in H$. Then

$$
\begin{gathered}
A\left(P_{M} x-P_{M}(A x)\right)=A\left(P_{M} x-P_{M}\left(A\left(P_{M} x+P_{M^{\perp}} x\right)\right)\right) \\
=A\left(P_{M} x-P_{M}\left(A\left(P_{M} x\right)\right)\right. \\
=0,
\end{gathered}
$$

which shows that $\left[A, P_{M}\right]=0$ for all $A \in a$. Thus $\left[a, P_{M}\right]=0$.

(iii) $\Rightarrow$ (ii): Suppose that $\left[a, P_{M}\right]=0$, and let $x \in M$ and $y \in M^{\perp}$. Then

$$
\langle A y, x\rangle=\left\langle A y, P_{M} x\right\rangle=\left\langle P_{M} A y, x\right\rangle=\left\langle A P_{M} y, x\right\rangle=0,
$$

for all $A \in a$ and hence $A\left(M^{\perp}\right) \subseteq M^{\perp}$, from where we conclude that $a\left(M^{\perp}\right) \subseteq M^{\perp}$.

We note that a subspace $M$ with either of the properties in Lemma 2.2 is called reducing (with respect to the subalgebra a).
We use Lemma 2.2 to state the Bicommutant/Double Commutant Theorem.

Theorem 2.3(von Neumann Double Commutant Theorem) Let $H$ be a Hilbert space and $a \subseteq B(H)$ be a unital selfadjoint *-subalgebra of $B(H)$. Then the following conditions are equivalent.

(i). $a=\{a\}$.

(ii). $a$ is closed with respect to the weak operator topology (WOT) on $B(H)$.

(iii). $a$ is closed with respect to the strong operator topology (SOT) on $B(H)$.

If a unital (self-adjoint) *-subalgebra $a$ of $B(H)$ satisfies either of the three equivalent conditions in Theorem 2.3, we say that it is a von Neumann algebra.

The Double Commutant Theorem simply asserts that the double commutant $\{a\}$ "of a unital self-adjoint subalgebra $a$ of $B(H)$ is always strongly closed (and hence weakly closed). That is, $a$ is strongly (and hence weakly) dense in $\{a\}^{\prime \prime}$. Equivalently, it says that the strongly closed unital selfadjoint subalgebras of $B(H)$ are always their own double commutant.

For convenience, we take a von Neumann algebra as a *subalgebra $a$ of $B(H)$ satisfying $a=\{a\} "$. A von Neumann algebra is a unital, weakly closed and contains an abundance of projections. If $a$ is a von Neumann algebra, then $a$ is generated by the projections in $a$.

Theorem 2.4 [12, Corollary 3.2.1] Let $T, S \in B(H)$. If $\operatorname{Lat}(T)=\operatorname{Lat}(S)$, then Hyperlat $(T)=\operatorname{Hyperlat}(S)$.

Proof. This follows easily from the definition.

Question 1. Does the condition that $\operatorname{Lat}(T)=\operatorname{Lat}(S)$ imply that $\{T\}^{\prime}=\{S\}^{\prime}$ ?

Question 2. Does the condition that $\{T\}^{\prime}=\{S\}^{\prime}$ imply that

$$
\operatorname{Hyperlat}(T)=\operatorname{Hyperlat}(S) \text { ? }
$$

We note that the converse of Theorem 2.4 need not hold in general. To see this, let $T=\left[\begin{array}{ll}0 & 1 \\ 0 & 0\end{array}\right]$ and $S=\left[\begin{array}{ll}0 & 0 \\ 0 & 1\end{array}\right]$ on the Hilbert space $H=\mathbb{R}^{2}$. A simple computation shows that

$$
\begin{aligned}
& \operatorname{Lat}(T)=\left\{\{0\},\left\langle\left(\begin{array}{l}
1 \\
0
\end{array}\right)\right\rangle, \mathbb{R}^{2}\right\} \\
& \neq\left\{\{0\},\left\langle\left(\begin{array}{l}
1 \\
0
\end{array}\right)\right\rangle,\left\langle\left(\begin{array}{l}
0 \\
1
\end{array}\right)\right\rangle, \mathbb{R}^{2}\right\}=\operatorname{Lat}(S) .
\end{aligned}
$$

Another computation shows that 
and

$$
\{S\}^{\prime}=\left\{\left[\begin{array}{cc}
b_{11} & 0 \\
0 & b_{22}
\end{array}\right]: b_{11}, b_{22} \in \mathbb{R}\right\} .
$$

We note that $\{T\}^{\prime} \cap\{S\}^{\prime}=\left\{\left[\begin{array}{cc}a_{11} & 0 \\ 0 & a_{11}\end{array}\right]: a_{11} \in \mathbb{R}\right\}$,

which is the set of scalar operators. Clearly the commutant of $T$ consists of operators similar to scalar operators. This result is true for isometries and co-isometries. Another computation shows that

$$
\begin{aligned}
& \operatorname{Hyperlat}(T)=\left\{\{0\},\left\langle\left(\begin{array}{l}
1 \\
0
\end{array}\right)\right\rangle, \mathbb{R}^{2}\right\} \\
& \neq\left\{\{0\},\left\langle\left(\begin{array}{l}
1 \\
0
\end{array}\right)\right\rangle,\left\langle\left(\begin{array}{l}
0 \\
1
\end{array}\right)\right\rangle, \mathbb{R}^{2}\right\}=\text { Hyperlat }(S) .
\end{aligned}
$$

However, it is clear that the subspace $M=\left\langle\left(\begin{array}{l}0 \\ 1\end{array}\right)\right\rangle \in \operatorname{Hyperlat}(T)$ if $a_{12}=0$.

This happens if and only if $\{T\}^{\prime}=\{\alpha I: \alpha \in \mathbb{C}\}$. This extra condition then implies that

$$
\text { Hyperlat }(T)=\text { Hyperlat }(S) \text {. }
$$

Theorem 2.4 can be relaxed as follows.

Corollary 2.5 [12, Corollary 3.2.2] Let $T, S \in B(H)$. If $\operatorname{Lat}(T) \equiv \operatorname{Lat}(S)$, then Hyperlat $(T) \equiv$ Hyperlat $(S)$.

Question 3. When is the converse of Corollary 2.5 true?

Let $T \in B(H)$. We define $W^{*}(T)$ to be the von Neumann algebra generated by $\{I, T\}$. Note that $W^{*}(T)=\{T\}^{\prime \prime} \cup\{\alpha I: \alpha \in \mathbb{C}\}$.

From the Double Commutant Theorem, if $T=T^{*}$, then $\{T\}^{\prime \prime}=W^{*}(T)$ and $\{T\}^{\prime}$ is a von Neumann algebra and is therefore generated by its projections. Since the projections in $\{T\}^{\prime}$ are also in $\left\{T^{*}\right\}^{\prime}$, it follows that the Double Commutant Theorem has the following reformulation.

$$
W^{*}(T)=\left\{T: P T=T P \text {, for every projection } P \in\{T\}^{\prime}\right\} .
$$

Corollary 2.6 Let $T \in B(H)$. Then $\operatorname{Lat}(T)=\operatorname{Lat}\left(W^{*}(T)\right)$.

Proof. Since $T \in W^{*}(T)$, trivially $\operatorname{Lat}\left(W^{*}(T)\right) \subseteq \operatorname{Lat}(T)$. On the other hand, $W^{*}(T)$ consists of polynomials in $I$ and $T$, and hence $\operatorname{Lat}(T) \subseteq \operatorname{Lat}\left(W^{*}(T)\right)$. Combining these two inclusions, equality follows. This proves the claim.
Corollary 2.7
Let $T \in B(H)$.
Then

Hyperlat $(T)=\operatorname{Lat}\left(\{T\}^{\prime}\right)$.

Theorem 2.8 Let $T \in B(H)$ and $M \in \operatorname{Hyperlat}(T)$. Then the orthogonal projection $P_{M}$ of $H$ onto $M$ belongs to $W^{*}(T)$.

Proof. By Theorem 2.3(Double Commutant Theorem), it suffices to show that if

$$
\begin{gathered}
Q=Q^{2}=Q^{*} \in\left\{W^{*}(T)\right\}^{\prime}=\{T\}^{\prime} \cap\left\{T^{*}\right\}^{\prime} \\
=\{L \in B(H): L T=T L\} \cap\left\{L \in B(H): T^{*} L=L T^{*}\right\} \\
=\left\{L \in B(H): L T=T L \text { and } T^{*} L=L T^{*}\right\},
\end{gathered}
$$

then $\left[P_{M}, Q\right]=0$, which says that $Q M \subseteq M$. Since $Q \in\{T\}^{\prime}$ and $M \in \operatorname{Hyperlat}(T)$, we have that $P_{M} \in W^{*}(T)$.

Remark. Let $T \in B(H)$. We call the set $\left\{W^{*}(T)\right\}$ the *commutant of $T$ and the set $\left\{W^{*}(T)\right\}^{\prime \prime}=\left\{\left\{W^{*}(T)\right\}^{\prime}\right\}^{\prime}$ the *bicommutant of $T$.

Theorem 2.8 helps us prove the following result.

Theorem 2.9 Let $A, B \in B(H)$. If $A \in W^{*}(B)$, then $\operatorname{Lat}(B) \subseteq \operatorname{Lat}(A)$.

Proof. We know that Hyperlat $(T) \subseteq \operatorname{Lat}(T)$ for any $T \in B(H)$ since $T$ commutes with itself (see [4]), that is, $T \in\{T\}^{\prime}$. Since $A \in W^{*}(B)$, we have that $Q P_{M}=P_{M} Q$, where $Q \in\left\{W^{*}(B)\right\}^{\prime}=\{B\}^{\prime} \cap\left\{B^{*}\right\}^{\prime} \quad$ is an orthogonal projection in $\{B\}^{\prime}$ and $M \in \operatorname{Hyperlat}(B)$, and hence $P_{M} A P_{M}=P_{M} A$, where $P_{M} \in W^{*}(A)$ is an orthogonal projection of $H$ onto $M$. This means that

$$
M \in \operatorname{Hyperlat}(B) \subseteq \operatorname{Lat}(B) \Rightarrow M \in \operatorname{Lat}(A) .
$$

Thus, $M \in \operatorname{Lat}(B) \Rightarrow M \in \operatorname{Lat}(A)$. This proves the claim. The converse of Theorem 2.9 is not true in general. However, if in addition $A B=B A$, then the converse is true.

Corollary 2.10 Let $A, B \in B(H)$. If $A \in W^{*}(B)$, then Hyperlat $(B) \subseteq$ Hyperlat $(A)$.

Proof. This follows from the proof of Theorem 2.9 and the fact that Hyperlat $(T) \subseteq \operatorname{Lat}(T)$ for any $T \in B(H)$.

Note that if $A \in\{T\}^{\prime}$, then $\overline{\operatorname{Ran}(A)}, \operatorname{Ker}(A) \in \operatorname{Lat}(T)$.

In addition, if $B \in\{T\}^{\prime \prime}, \quad$ then $\overline{\operatorname{Ran}(A)}$, $\operatorname{Ker}(A) \in \operatorname{Hyperlat}(T)$.

A lattice $L$ of subspaces of a Hilbert space $H$ is said to be trivial if $L=\{\{0\}, H\}$.

Proposition 2.11[12] Let $A, B, T \in B(H)$. If $T$ doubly intertwines $A$ and $B$ and $\operatorname{Lat}(A) \cap \operatorname{Lat}(B)$ is trivial, then either $T=0$ or $T$ is a quasiaffinity.

Proof. Suppose $T$ doubly intertwines the pair $(A, B)$. Then 
$T A=B T$ and $T B=A T$. Since $T A=B T$, we have $\overline{\operatorname{Ran}(T)} \in \operatorname{Lat}(B)$ and $\operatorname{Ker}(T) \in \operatorname{Lat}(A)$. Since $T B=A T$, we deduce that

$$
\overline{\operatorname{Ran}(T)} \in \operatorname{Lat}(A) \bigcap \operatorname{Lat}(B)
$$

and

$$
\operatorname{Ker}(T) \in \operatorname{Lat}(A) \bigcap \operatorname{Lat}(B) .
$$

If $\overline{\operatorname{Ran}(T)}=\{0\}$, then $T=0$. If $\overline{\operatorname{Ran}(T)}=H$, then $\operatorname{Ker}(T)=\{0\}$ and hence $T$ is injective with dense range, and therefore a quasiaffinity.

Proposition 2.11 can be strengthened into the following results.

Corollary 2.12 Let $A, B, T \in B(H)$. If $T$ commutes with $A$ and $B$ and $\operatorname{Lat}(A) \bigcap \operatorname{Lat}(B)$ is trivial, then either $T=0$ or $T$ is a quasiaffinity.

Proof. If $T$ commutes with $A$ and $B$, then $T A=A T$ and $T B=B T$. Using Proposition 2.8, we have

$$
\overline{\operatorname{Ran}(T)} \in \operatorname{Lat}(A) \bigcap \operatorname{Lat}(B)
$$

and

$$
\operatorname{Ker}(T) \in \operatorname{Lat}(A) \bigcap \operatorname{Lat}(B) .
$$

By the same argument then either $T=0$ or $T$ is a quasiaffinity.

Corollary 2.13 Let $A, T \in B(H)$. If $T$ commutes with $A$ and $\operatorname{Lat}(A)$ is trivial, then either $T=0$ or $T$ is a quasiaffinity.

Proof. If $T$ commutes with $A$ then $T A=A T$. Using Corollary 2.9, we have $\overline{\operatorname{Ran}(T)} \in \operatorname{Lat}(A) \quad$ and $\operatorname{Ker}(T) \in \operatorname{Lat}(A)$. Triviality of $\operatorname{Lat}(A)$ then implies that $\overline{\operatorname{Ran}(T)}=\{0\}$, and thus $T=0$. If $\overline{\operatorname{Ran}(T)}=H$, then $\operatorname{Ker}(T)=\{0\}$, which proves that $T$ is a quasiaffnity.

\section{Invariant Subspace Lattice Operations}

Theorem 3.1 Let $T \in B(H)$. Then $M \in \operatorname{Lat}(T)$ if and only if $M^{\perp} \in \operatorname{Lat}\left(T^{*}\right)$.

Clearly the map $\varphi: M \rightarrow M^{\perp}$ of $\operatorname{Lat}(T)$ into $\operatorname{Lat}\left(T^{*}\right)$ is a (lattice) isomorphism. This map "inverts" the lattice operations:

$$
{\underset{\alpha}{\vee}}_{\alpha} \mapsto{ }_{\alpha} M_{\alpha}^{\perp}
$$

and

$$
\hat{\alpha}_{\alpha} \mapsto \underset{\alpha}{\vee} M_{\alpha}{ }^{\perp}
$$

\section{Operator Equivalences and Lattices}

Recall that if $L_{1}$ and $L_{2}$ are lattices of subspaces of a Hilbert space $H$, an isomorphism $\varphi: L_{1} \rightarrow L_{2}$ is a one-toone and onto map with the property that if $M_{1}, M_{2} \in L_{1}$ then $M_{1} \subseteq M_{2}$ if and only if $\varphi\left(M_{1}\right) \subseteq \varphi\left(M_{2}\right)$.

In this paper, lattice refers to either Lat $(T), \operatorname{Hyperlat}(T)$, or $\operatorname{Re} d(T)$.

In this section we investigate lattices of operators in some equivalence classes emanating from certain operator equivalence relations.

Theorem 4.1 Similarity of operators preserves non-trivial invariant and non-trivial hyperinvariant subspaces.

Proof. We prove the case for invariance. The proof for hyperinvariance can be proved similarly. Suppose $A, B \in B(H)$ are such that $A=X^{-1} B X$. That is, $X A=B X$. Suppose $M$ is a non-trivial $A$-invariant subspace. Then $B X M=X A M \subseteq X M$.

Since $M$ is non-trivial and $X$ is invertible, we conclude that $X M$ is a non-trivial invariant subspace for $B$. Thus $M$ is $A$-invariant if and only if $M$ is $B$-invariant.

It has been proved (see [5], [6]) that if $A$ and $B$ are quasisimilar and one has a nontrivial hyperinvariant subspace, then so does the other. However, similar (quasisimilar) operators need not have isomorphic invariant (hyperinvariant) lattices. An example is given in Herrero[5] of two quasisimilar nilpotent operators of the same order but with non-isomorphic hyperlattices. This shows that structure of the hyperlattice of an operator is not preserved under quasisimilarity.

Example 1. Let $A=\left[\begin{array}{ll}0 & 1 \\ 0 & 1\end{array}\right]$ and $B=\left[\begin{array}{ll}0 & 0 \\ 0 & 1\end{array}\right]$. A simple computation shows that $A$ and $B$ are similar. However, another computation shows that $\operatorname{Lat}(A)=\left\{\{0\},\left\langle\left(\begin{array}{l}1 \\ 0\end{array}\right),, \mathbb{R}^{2}\right\}\right.$ and

$$
\operatorname{Lat}(B)=\left\{\{0\},\left\langle\left(\begin{array}{l}
1 \\
0
\end{array}\right)\right\rangle,\left\langle\left(\begin{array}{l}
0 \\
1
\end{array}\right)\right\rangle, \mathbb{R}^{2}\right\}
$$

Clearly $\operatorname{Lat}(A)$ and $\operatorname{Lat}(B)$ are not isomorphic.

Theorem 4.2 If $T_{1}, T_{2} \in B(H)$ are quasisimilar with quasiaffinities $X$ and $Y$, then $X Y \in\left\{T_{1}\right\}^{\prime}$ and $Y X \in\left\{T_{2}\right\}^{\prime}$.

Proof. Suppose $T_{1} X=X T_{2}$ and $T_{2} Y=Y T_{1}$, where $X$ and $Y$ are quasiaffinities. Post-multiplying the first equation by $Y$ and using the second equation, we have

$$
T_{1} X Y=X T_{2} Y=X Y T_{1},
$$

which proves that $X Y \in\left\{T_{1}\right\}^{\prime}$. Post-multiplying the second equation by $X$ and using the first equation we have 


$$
T_{2} Y X=Y T_{1} X=Y X T_{2}
$$

which proves that $Y X \in\left\{T_{2}\right\}^{\prime}$.

Theorem 4.3 Hyper-quasi-similarity is an equivalence relation.

Proof. Suppose $T_{1}, T_{2} \in B(H)$ are quasisimilar and there exists an implementing pair $(X, Y)$ of quasiaffinities such that $X Y$ has the hereditary property with respect to $T_{1}$ and $Y X$ has the hereditary property with respect to $T_{2}$. Let $M_{1} \in \operatorname{Hyperlat}\left(T_{1}\right)$ and $M_{2} \in \operatorname{Hyperlat}\left(T_{2}\right)$.Then

$$
X T_{1}=T_{2} X, T_{1} Y=Y T_{2} \text { and } \overline{Y X M}_{1}=M_{1} \text { and } \overline{X Y M}_{2}=M_{2} \text {. }
$$

Without loss of generality, if we let $T_{1}=T_{2}$, then we have $X T_{1}=T_{1} X, T_{1} Y=Y T_{1}$ and $\overline{Y X M}_{1}=M_{1}$ and $\overline{X Y M}_{2}=M_{2}$. From Theorem 4.2, we know that both $X Y$ and $Y X$ are quasiaffinities and $X Y, Y X \in\left\{T_{1}\right\}^{\prime}$. Thus, $\overline{Y X M}_{1}=M_{1}$ and $\overline{X Y M}_{2}=M_{2}$, where $M_{1}, M_{2} \in \operatorname{Hyperlat}\left(T_{1}\right)$. This proves reflexivity of $\stackrel{h}{\approx}$.

Now suppose $T_{1} \stackrel{h}{\approx} T_{2}$. Then by re-writing the definition above, we have $Y T_{2}=T_{1} Y, T_{2} X=X T_{1}$ and $\overline{X Y M_{2}}=M_{2}$ and $\overline{Y X M}_{1}=M_{1}, \quad$ where $\quad M_{1} \in \operatorname{Hyperlat}\left(T_{1}\right) \quad$ and $M_{2} \in \operatorname{Hyperlat}\left(T_{2}\right)$. Thus $T_{2} \stackrel{h}{\approx} T_{1}$. This proves the symmetry property of $\stackrel{h}{\approx}$.

Now suppose $T_{1} \stackrel{h}{\approx} T_{2}$ (with implementations as above) and suppose also that $T_{2} \stackrel{h}{\approx} T_{3}$, for some $T_{3} \in B(H)$. Then there exists an implementing pair of quasiaffinities $(Z, S)$ such that $Z T_{2}=T_{3} Z, T_{2} S=S T_{3}$ and $\overline{S Z M_{2}}=M_{2}$ and $\overline{Z S M_{3}}=M_{3}$, where $\quad M_{2} \in \operatorname{Hyperlat}\left(T_{2}\right) \quad$ and $\quad M_{3} \in \operatorname{Hyperlat}\left(T_{3}\right) . \quad \mathrm{A}$ simple computation shows that

$$
\begin{aligned}
& Z X T_{1}=T_{3} Z X, S Y T_{1}=T_{3} S Y, \\
& T_{1} X Z=X Z T_{3}, T_{1} Y S=Y S T_{3}, \\
& Z S X Y \in\left\{T_{2}\right\}^{\prime}, Y S Z X \in\left\{T_{1}\right\}^{\prime}, \\
& X Y S Z \in\left\{T_{2}\right\}^{\prime}, Z X Y S \in\left\{T_{3}\right\}^{\prime} .
\end{aligned}
$$

This is equivalent to

$$
\overline{Y S Z X M_{1}}=M_{1}
$$

And

$$
\overline{\operatorname{ZXYSM}_{3}}=M_{3}, M_{1} \in \operatorname{Hyperlat}\left(T_{2}\right)
$$

and $M_{3} \in \operatorname{Hyperlat}\left(T_{3}\right)$. This proves that $T_{1} \approx T_{3}$, and hence $h$ $\approx$ is transitive.

Theorem 4.4 ([8], Corollary 4.8) Hyper-quasi-similarity preserves nontrivial hyperinvariant invariant subspaces.

Proof. This follows easily from the fact that hypersimilarity is stronger than quasisimilarity and the fact that quasisimilarity preserves non-trivial hyperinvariant subspaces.

Kubrusly in [8] has shown that non-scalar normal operators have non-trivial hyperinvariant subspaces. Thus Theorem 4.4 ensures that an operator quasisimilar to a nonscalar normal operator has a non-trivial hyperinvariant subspace.

The following result is a strengthening of ([8], Lemma 4.7) and lends credence to Theorem 4.4.

Theorem 4.5 Let $T \in B(H)$ be quasisimilar to a unitary operator $U \in B(K)$ and let $M \subseteq K$. If $M \in \operatorname{Hyperlat}(T)$ then $M \in \operatorname{Hyperlat}(U)$.

Proof. Suppose $T X=X U$ and $U Y=Y T$, where $X \in B(K, H), Y \in B(H, K)$ are quasiaffinities. If $A \in\{T\}^{\prime}$, then

$$
\begin{aligned}
U(Y A X)= & (U Y)(A X)=(Y T)(A X) \\
& =Y(T A) X \\
& =(Y A)(T X) \\
& =(Y A)(X U) \\
& =(Y A X) U
\end{aligned}
$$

This proves that $Y A X \in\{U\}^{\prime}$ for every $A \in\{T\}^{\prime}$.

Using the computation, we conclude that $M$ is invariant for $Y A X$, and hence for any operator that commutes with $U$. This proves the claim.

Corollary 4.6 If $T \in B(H)$ is quasisimilar to a unitary operator $U \in B(K)$ then Hyperlat $(T) \subseteq \operatorname{Re} d(U)$.

Proof. Follows from the fact that for a unitary operator $U$, $\operatorname{Hyperlat}(U)=\operatorname{Re} d(U)$.

Theorem 4.7 Let $T, A \in B(H)$ such that $T A=A T$. If $M \in \operatorname{Hyperlat}(T)$ then $M \in \operatorname{Re} d(T)$.

Proof. From the hypothesis and definition, it follows that $M \in \operatorname{Hperlat}(A) \subseteq \operatorname{Lat}(A)$, and hence $M$ reduces $\{A\}^{\prime}$. In particular $M$ reduces $T$.

Theorem 4.8 Let $A \in B(H)$ and $B \in B(K)$ be self-adjoint operators. If there exists a quasiaffinity $X \in B(H, K)$ such that $X A=B X$, then $A$ and $B$ are unitarily equivalent.

Theorem 4.8 has been extended to the class of normal operators as a consequence of ([9], Corollary 6.50).

The following result gives a condition when some subspace lattices for two operators are isomorphic.

Theorem 4.9 Suppose that $T \in B(H)$, where $H$ is a finite 
dimensional Hilbert space and $\varphi: B(H) \rightarrow B(H)$ defined by $T \rightarrow \varphi(T)$ is a linear map. Then the following statements are equivalent.

(i). $\operatorname{Lat}(T) \equiv \operatorname{Lat}(\varphi(T))$.

(ii). Hyperlat $(T) \equiv$ Hyperlat $(\varphi(T))$.

(iii). $\operatorname{Re} d(T) \equiv \operatorname{Re} d(\varphi(T))$.

From Theorem 4.9, we can conclude that

$$
\operatorname{Lat}(T) \equiv \operatorname{Lat}(c T), \text { Hyperlat }(T) \equiv \text { Hyperlat }(c T)
$$

and

$$
\operatorname{Re} d(T) \equiv \operatorname{Re} d(c T)
$$

where $0 \neq c \in \mathbb{C}$.

\section{Reducibility and Subspace Lattices}

It is clear that reducing subspaces are generally easier to treat that arbitrary invariant subspaces.

Theorem 5.1 A subspace $M$ reduces an operator $T$ if and only if $M \in \operatorname{Lat}(T) \cap \operatorname{Lat}\left(T^{*}\right)$.

Proof. Follows easily from the definition.

Corollary 5.2 Let $T \in B(H)$ and $M$ be a subspace of $H$. The following statements are equivalent.

(i). $M$ reduces $T$.

(ii). $M \in \operatorname{Lat}(T) \cap \operatorname{Lat}\left(T^{*}\right)$.

(iii). $P_{M} \in\{T\}^{\prime}$, where $P_{M}$ is the orthogonal projection of $H$ onto $M$.

Theorem 5.3 If $T_{1} \in B\left(H_{1}\right)$ and $T_{2} \in B\left(H_{2}\right)$ are irreducible, then every operator $A \in B\left(H_{1}, H_{2}\right)$ that intertwines them is either zero or identity.

Theorem 5.4 If an operator $A$ commutes with an irreducible operator $T$, then $A$ is similar to a scalar operator.

Theorem 5.5 If $T \in B(H)$ is nilpotent of nil-index $n$, then $\operatorname{Re} d(T)=\{\{0\}, H\}$.

Corollary 5.6 Let $T \in B(H)$. If $\operatorname{Re} d(T)=\{\{0\}, H\}$, then $T=\alpha I+S$, where $S$ is a nilpotent operator.

Bercovici etal[1] have proved that for a nilpotent operator $T \in B(H)$ such that $T^{n}=0$, for some integer $n \geq 1$, $\operatorname{Hyperlat}(T)$ is generated by the spaces $\operatorname{Ker}\left(T^{m}\right)$ and $\operatorname{Ran}\left(T^{m}\right), m=0,1,2,3, \cdots, n$. They have also shown that $\operatorname{Ran}\left(T^{n-1}\right)$ is the smallest non-trivial hyperinvariant subspace and that $\operatorname{Ker}\left(T^{n-1}\right)$ is the largest non-trivial hyperinvariant subspace.

We show the relationship between Hyperlat(T) and $\operatorname{Re} d(T)$, where $T$ is a unitary operator.

Theorem 5.7 Let $T \in B(H)$ be a unitary operator. A subspace $M \subseteq H$ is hyperinvariant for $T$ if and only if $M$ reduces $T$.
Proof. Suppose that $M \in$ Hyperlat $(T)$ and let $P_{M}$ be the orthogonal projection of $H$ onto $M$. Then $A P_{M}=P_{M} A P_{M}$ for every $A \in\{T\}^{\prime}$. Since $T$ is unitary and hence normal, by Fuglede's theorem, $A^{*} \in\{T\}^{\prime}$. Thus $A^{*} P_{M}=P_{M} A^{*}$ and hence $A P_{M}=P_{M} A P_{M}=P_{M} A$. By Corollary 5.2, we have that $M$ reduces $T$. Conversely, suppose $M$ reduces $T$. Without loss of generality, suppose $A P_{M}=P_{M} A$. Then

$$
A M=A P_{M} H=P_{M} A H \subseteq P_{M} H=M .
$$

This shows that $M$ is invariant under $A$.

So, if $A P_{M}=P_{M} A$ for all $A \in\{T\}^{\prime}$, then $M$ is hyperinvariant for $T$.

Remark. Theorem 5.7 says that for a unitary operator $T$, Hyperlat $(T)=\operatorname{Re} d(T)$.

Theorem 5.7 can be relaxed as follows.

Theorem 5.8 Let $T \in B(H)$ be an isometry. If $M \subseteq H$ is such that $T M=M$ then $M$ reduces $T$.

Proof. If $T M=M$ then $T^{*} M=T^{*} T M=M$. This proves the claim.

Corollary 5.9 Let $T \in B(H)$ be an isometry. If $M \subseteq H$ is such that $T M=M$ then $\operatorname{Re} d(T)=\operatorname{Lat}(T)$.

Proof. This follows from Theorem 5.8 and the fact that $\operatorname{Re} d(T) \subseteq \operatorname{Lat}(T)$, for any operator $T$.

Let $T \in B(H)$. If a subspace $M \subseteq H$ reduces every operator in the commutant of $T$, then we say that $M$ is a hyper-reducing subspace for $T$.

Example 2. A unilateral shift $S: \ell^{2}(\mathbb{N}) \rightarrow \ell^{2}(\mathbb{N})$ of multiplicity one is irreducible and so is its two-dimensional analogue. The operator

$$
A=\left[\begin{array}{ll}
0 & 0 \\
1 & 0
\end{array}\right]
$$

is the two-dimensional analogue of the unilateral shift operator of multiplicity one. We note that

$$
\operatorname{Lat}(S)=\left\{\{0\},\left\langle\left(\begin{array}{l}
0 \\
1
\end{array}\right)\right\rangle, \mathbb{R}^{2}\right\} \text {. A simple computation }
$$

shows that $M=\left\langle\left(\begin{array}{l}0 \\ 1\end{array}\right)\right\rangle \in$ Hyperlat $(S)$ and that

$M \notin \operatorname{Re} d(S)$. So $S$ has no non-trivial reducing subspace and hence it is irreducible. Note also, that $\operatorname{Lat}(S) \neq \operatorname{Lat}\left(S^{*}\right)$ but $\operatorname{Lat}(S) \equiv \operatorname{Lat}\left(S^{*}\right)$.

The operator

$$
T=\left[\begin{array}{ccc}
1 & 0 & 0 \\
0 & 0 & 1 \\
0 & 1 & -1
\end{array}\right]=1 \oplus\left[\begin{array}{cc}
0 & 1 \\
1 & -1
\end{array}\right]
$$


is reducible in $H=\mathbb{R}^{3}$.

Clearly, $\quad T \in B(H)$ is irreducible if its commutant $\{T\}^{\prime}=\{A \in B(H): T A=A T\}$ contains no projections other 0 and the identity $I$ on $H$. That is, if there is no non-trivial orthogonal projection commuting with $T$. This is equivalent to saying that $\operatorname{Re} d(T)=\{\{0\}, H\}$. The class of irreducible operators is huge. In fact the class of irreducible operators is dense in $B(H)$ in the norm topology.

An operator $T \in B(H)$ is reductive if all its invariant subspaces reduce it.

There are several equivalent ways to characterize a reductive operator.

Note that $\operatorname{Re} d(T)=\operatorname{Re} d\left(T^{*}\right), \quad$ for any operator $T \in B(H)$.

Corollary 5.10 Let $T \in B(H)$. If $\operatorname{Lat}(T) \subseteq \operatorname{Lat}\left(T^{*}\right)$, then $T$ is reductive.

Corollary 5.11 An operator $T \in B(H)$ is reductive if and only if $\operatorname{Lat}(T)=\operatorname{Re} d\left(T^{*}\right)$.

Proof. By definition, if $T$ is reductive, then

$\operatorname{Lat}(T) \subseteq \operatorname{Re} d(T)=\operatorname{Re} d\left(T^{*}\right)$. But the inclusion $\operatorname{Re} d(T) \subseteq \operatorname{Lat}(T)$ is obvious. Combining these statements, we have equality. Conversely, suppose that

$$
\operatorname{Lat}(T)=\operatorname{Re} d\left(T^{*}\right) .
$$

Then

$$
\begin{gathered}
\operatorname{Lat}(T)=\operatorname{Re} d\left(T^{*}\right) \\
=\operatorname{Lat}\left(T^{*}\right) \bigcap \operatorname{Lat}(T) \\
\subseteq \operatorname{Lat}\left(T^{*}\right) .
\end{gathered}
$$

Thus $\operatorname{Lat}(T) \subseteq \operatorname{Lat}\left(T^{*}\right)$.

The class of reducible operators contains the class of reductive operators. However, an operator may be reducible but fail to be reductive. Thus,

\section{$\operatorname{Re}$ ductive $\subset \operatorname{Re}$ ducible.}

Note that every self-adjoint (and by extension, normal operator on a finite dimensional Hilbert space) is reductive. It is also known that every compact normal operator is reductive. It is a known fact that every operator that commutes with a non-scalar normal operator is reducible.

In fact for a normal operator $T \in B(H)$, we have that

$$
\operatorname{Lat}(T) \equiv \operatorname{Lat}\left(T^{*}\right) .
$$

Example 3. Let $T=\left[\begin{array}{lll}0 & 1 & 0 \\ 0 & 0 & 0 \\ 0 & 0 & 1\end{array}\right]$.
Then $T=\left[\begin{array}{ll}0 & 1 \\ 0 & 0\end{array}\right] \oplus 1$

and hence $T$ is reducible. A simple computation shows that

$$
\operatorname{Lat}(T)=\left\{\{0\},\left(\left(\begin{array}{l}
1 \\
0 \\
0
\end{array}\right)\right\rangle,\left\langle\left(\begin{array}{l}
0 \\
0 \\
1
\end{array}\right)\right\rangle,\left\langle\left(\begin{array}{l}
1 \\
0 \\
0
\end{array}\right),\left(\begin{array}{l}
0 \\
0 \\
1
\end{array}\right)\right), \mathbb{R}^{3}\right\}
$$

while

$$
\operatorname{Lat}\left(T^{*}\right)=\left\{\{0\},\left\langle\left(\begin{array}{l}
1 \\
0 \\
0
\end{array}\right)\right),\left\langle\left(\begin{array}{l}
0 \\
0 \\
1
\end{array}\right)\right\rangle, \mathbb{R}^{3}\right\} .
$$

Thus $\operatorname{Lat}(T) \neq \operatorname{Lat}\left(T^{*}\right)$. Therefore $T$ is not reductive.

A simple calculation shows that $T$ is not a normal operator.

Clearly,

Normal $\subset \operatorname{Re}$ ductive $\subset \operatorname{Re}$ ducible.

The above inclusion is strict. For instance it has been shown in [12] that not every reductive operator in normal. Moore[16] went further and gave some conditions under which a reductive operator is normal: that such a reductive operator $T$ must commute with an injective compact operator or $T$ is polynomially compact or $T$ is expressible as a sum of a normal operator and a commuting compact operator (see [16], Theorem 1 and Corollary 2).

Example 4. The bilateral shift $B$ on $\ell^{2}(\mathbb{Z})$ defined by $B\left(\cdots, x_{-2}, x_{-1},\left[x_{0}\right], x_{1}, x_{2}, \cdots\right)=\left(\cdots, x_{-2}, x_{-1}, x_{0}, x_{1}, x_{2}, \cdots\right)$, where $\quad x=\left(\cdots, x_{-2}, x_{-1},\left[x_{0}\right], x_{1}, x_{2}, \cdots\right) \in \ell^{2}(\mathbb{Z}) \quad$ and $\quad\left[x_{0}\right]$ denotes the 0 -th coordinate of $x$, is not reductive.

Indeed,

$$
\begin{gathered}
M=\left\{x \in \ell^{2}(\mathbb{Z}): x_{n}=0, \text { if if } n<0\right\} \in \operatorname{Lat}(B) \text { but } \\
M \notin \operatorname{Lat}\left(B^{*}\right) .
\end{gathered}
$$

Theorem 5.12 A reductive operator is normal if and only if it has a non-trivial invariant subspace.

Theorem 5.13 Let $T \in B(H)$. If a subspace $M \subseteq H$ is hyper-reducing then $M \in \operatorname{Lat}\left(\{T\}^{\prime}\right) \cap \operatorname{Lat}\left(\left\{T^{*}\right\}^{\prime}\right)$.

Example 5. Let $A=\left[\begin{array}{ll}1 & 0 \\ 0 & 1\end{array}\right]$ and $B=\left[\begin{array}{ll}1 & 1 \\ 0 & 1\end{array}\right]$.

Clearly these two operators are not similar. A simple computation shows that $\operatorname{Lat}(A)=\left\{\{0\},\left\langle\left(\begin{array}{l}1 \\ 0\end{array}\right)\right\rangle,\left\langle\left(\begin{array}{l}0 \\ 1\end{array}\right)\right\rangle, \mathbb{R}^{2}\right\}=\operatorname{Re} d(A)$, and 


$$
\operatorname{Lat}(B)=\left\{\{0\},\left\langle\left(\begin{array}{l}
1 \\
0
\end{array}\right)\right\rangle, \mathbb{R}^{2}\right\} \neq\left\{\{0\}, \mathbb{R}^{2}\right\}=\operatorname{Re} d(A) .
$$

Thus $A$ is reductive while $B$ is not since not every invariant subspace of $B$ reduces $B$. Another computation shows that

$$
\{B\}^{\prime}=\left\{X: X=\left[\begin{array}{cc}
\alpha & \beta \\
0 & \alpha
\end{array}\right], \alpha, \beta \in \mathbb{R}\right\}
$$

and

$$
\{A\}^{\prime}=\left\{Y: Y=\left[\begin{array}{cc}
\alpha & \beta \\
\gamma & \lambda
\end{array}\right], \alpha, \beta, \gamma, \lambda \in \mathbb{R}\right\}
$$

hence

$$
\text { Hyperlat }(A)=\left\{\{0\}, \mathbb{R}^{2}\right\}
$$

and

$$
\text { Hyperlat }(B)=\operatorname{Lat}(B) \text {. }
$$

Theorem 5.14 ([13], Theorem $\mathrm{H}$ ) If $A$ is a reductive operator then $A$ can be written as a direct sum $A=A_{1} \oplus A_{2}$ where $A_{1}$ is normal, $A_{2}$ is reductive, $\{A\}^{\prime}=\left\{A_{1}\right\}^{\prime} \oplus\left\{A_{2}\right\}^{\prime}$, and all the invariant subspaces of $A_{2}$ are hyperinvariant.

Corollary 5.15 [6] Suppose $A$ is a reductive operator such that $A=A_{1} \oplus A_{2}$. Then

$$
\operatorname{Hyperlat}(A)=\operatorname{Hyperlat}\left(A_{1}\right) \oplus \operatorname{Hyperlat}\left(A_{2}\right)
$$

and

$$
\operatorname{Lat}(A)=\operatorname{Hyperlat}(A) .
$$

From Theorem 5.14 and Corollary 5.15 we conclude that if $A$ is reductive and completely non-normal (that is, $A$ has no normal direct summand) then $\operatorname{Lat}(A)=\operatorname{Lat}\left(\{A\}^{\prime}\right)$.

Theorem 5.16 ([13], Corollary 1) If $A$ is a reductive operator, then every hyperinvariant subspace of $A$ is hyperreducing.

Corollary 5.17 If $A$ is a reductive operator, then Hyperlat $(A) \subseteq$ Hyper $\operatorname{Re} d(A)$.

Corollary 5.17 says that if $A$ is reductive then $\operatorname{Lat}\left(\{A\}^{\prime}\right)=\operatorname{Lat}\left(\left\{A^{*}\right\}^{\prime}\right)$.

Theorem 5.18 Let $T \in B(H)$. Then

$$
\text { Hyper } \operatorname{Re} d(T)=\operatorname{Lat}\left(\{T\}^{\prime}\right) \cap \operatorname{Lat}\left(\left\{T^{*}\right\}^{\prime}\right) \text {. }
$$

Proof.

$$
\text { Hyper } \operatorname{Re} d(T)=\left\{M \subseteq H: M \in \operatorname{Re} d\left(\{T\}^{\prime}\right)\right\}
$$

$$
\begin{gathered}
=\left\{M \subseteq H: S M \subseteq M, S^{*} M \subseteq M, S \in\{T\}^{\prime}\right\} \\
=\left\{M \subseteq H: M \in \operatorname{Lat}(S) \cap \operatorname{Lat}\left(S^{*}\right), S \in\{T\}^{\prime}\right\} \\
=\left\{M \subseteq H: M \in \operatorname{Lat}\left(\{T\}^{\prime}\right) \cap \operatorname{Lat}\left(\left\{T^{*}\right\}^{\prime}\right)\right\} \\
=\operatorname{Lat}\left(\{T\}^{\prime}\right) \cap \operatorname{Lat}\left(\left\{T^{*}\right\}^{\prime}\right) .
\end{gathered}
$$

Theorem 5.19 Let $T \in B(H)$. Then

$$
\text { Hyper } \operatorname{Re} d(T)=\text { Hyperlat }(T) \cap \text { Hyperlat }\left(T^{*}\right) .
$$

Proof. The proof follows from Theorem 5.18 and the fact that

$$
\operatorname{Lat}\left(\{T\}^{\prime}\right)=\operatorname{Hyperlat}(T)
$$

and

$\operatorname{Lat}\left(\left\{T^{*}\right\}^{\prime}\right)=\operatorname{Hyperlat}\left(T^{*}\right)$, for any $T \in B(H)$.

Corollary 5.20 Let $T \in B(H)$ be self-adjoint. Then

$$
\text { Hyper } \operatorname{Re} d(T)=\text { Hyperlat }(T) \text {. }
$$

Proof. The proof follows easily from Theorem 5.19 and the fact that self-adjointness of $T$. The proof also follows from Theorem 5.18, the self-adjointness of $T$ and the fact that $\operatorname{Lat}\left(\{T\}^{\prime}\right)=\operatorname{Hyperlat}(T)$.

Proposition 5.21([1], Proposition 2.2) Let $T \in B(H)$ be normal. Then Hyperlat $(T)=\left\{M \subseteq H: P_{M} \in W^{*}(T)\right\}$.

Corollary 5.22 Let $T \in B(H)$ be normal. Then every hyperinvariant subspace of $T$ is hyperinvariant for $T^{*}$.

Corollary 5.22 says Hyperlat $(T) \subseteq \operatorname{Hyperlat}\left(T^{*}\right)$, for any normal operator $T \in B(H)$.

The converse of Corollary 5.22 is also true. This leads to the following result.

Corollary 5.23 Let $T \in B(H)$ be normal. Then $\operatorname{Hyperlat}(T)=\operatorname{Hyperlat}\left(T^{*}\right)$.

Proof. Since $T$ is normal if and only if $T^{*}$ is normal, the result follows from the fact that $T^{*} \in\{T\}^{\prime}$ if and only if $T \in\left\{T^{*}\right\}^{\prime}$

Theorem 5.24 If $T \in B(H)$ is an invertible reductive operator, then $T^{-1}$ is also reducible.

Proof. Since $T \in B(H)$ is reducible, by Theorem 5.14 it can be expressed as

$$
T=\left[\begin{array}{cc}
T_{1} & 0 \\
0 & T_{2}
\end{array}\right]=T_{1} \oplus T_{2},
$$

with respect to the direct sum decomposition $H=M \oplus M^{\perp}$, where $M$ is a subspace that reduces $T$. Invertibility of $T$ 
implies that of $T_{1}$ and $T_{2}$. Thus

$$
T^{-1}=\left[\begin{array}{cc}
T_{1}^{-1} & 0 \\
0 & T_{2}^{-1}
\end{array}\right]=T_{1}^{-1} \oplus T_{2}^{-1},
$$

with respect to the direct sum decomposition $H=M \oplus M^{\perp}$.

Corollary 5.25 Let $T \in B(H)$ be invertible. If a subspace $M \subseteq H$ reduces $T$, then $M$ reduces $T^{-1}$.

Proof. Let $P_{M}$ be the orthogonal projection of $H$ onto $M$. Since $M$ reduces $T$, we have $T P_{M}=P_{M} T$. By the proof of Theorem 5.24, $T^{-1} P_{M}=P_{M} T^{-1}$. This proves the claim.

Remark. From Theorem 5.24 and Corollary 5.25, we conclude that if $T \in B(H)$ is invertible, then $\operatorname{Re} d(T)=\operatorname{Re} d\left(T^{-1}\right)$.

We also note that Corollary 5.25 is not true if we replace reducibility with invariance. This is because in infinite dimensional Hilbert spaces, the invariance of a subspace $M$ for an invertible operator $T \in B(H)$ does not imply invariance under $T^{-1}$.

Example 6. Consider the bilateral weighted shift $T_{\omega}$ on $\ell^{2}(\mathbb{Z})$ defined by $T_{\omega} e_{n}=\omega_{n} e_{n+1}$, where $n \in \mathbb{Z}$ and $\left\{e_{n}\right\}$ the canonical orthonormal basis for $\ell^{2}(\mathbb{Z})$. A simple calculation shows that $T_{\omega}^{-1} e_{n}=\frac{1}{\omega_{n}} e_{n}$, where $n \in \mathbb{Z}$. If

$M=\operatorname{span}\left\{e_{1}, e_{2}, \cdots\right\}$, then $M$ is invariant for $T_{\omega}$ but is not invariant for $T_{\omega}{ }^{-1}$.

The following result shows that taking powers of an operator $T \in B(H)$ preserves invariance and reduction.

Theorem 5.26. Let $T \in B(H)$ and $M \subseteq H$. The following statements are true for any integer $n>1$.

(i). If $M \in \operatorname{Lat}(T)$ then $M \in \operatorname{Lat}\left(T^{n}\right)$.

(ii). If $M \in \operatorname{Re} d(T)$ then $M \in \operatorname{Re} d\left(T^{n}\right)$.

Proof. The proofs of (i) and (ii) follow easily by mathematical induction on $n \in \mathbb{N}$. In the proof of (ii), we use the fact that $M \in \operatorname{Re} d(T)$ implies that $T M \subseteq M$ and $T^{*} M \subseteq M$.

Theorem 5.27. Let $T \in B(H)$ and $M \subseteq H$. If $M \in \operatorname{Hyperlat}(T)$ then $M \in \operatorname{Hyperlat}\left(T^{n}\right)$, for any integer $n>1$.

Proof. We need to prove that $M \in \operatorname{Lat}(S)$, where $S \in\{T\}^{\prime}$ implies that $M \in \operatorname{Lat}(X)$, where $X \in\left\{T^{n}\right\}^{\prime}$.

By Theorem 5.26(i), if $M \in \operatorname{Lat}(S)$ then $M \in \operatorname{Lat}\left(S^{n}\right)$, where $S \in\{T\}^{\prime}$.

By mathematical induction on $n \in \mathbb{N}$, if $S \in\{T\}^{\prime}$ then
$S^{n} \in\{T\}^{\prime}, T^{n} \in\{S\}^{\prime}$ and $S^{n} \in\left\{T^{n}\right\}$.

By letting $X=S^{n}$, and using Theorem 5.26(i) once more, the result follows.

\section{Discussion}

The invariant subspaces of an operator, their classification and description play an explicitly central role in operator theory. They are a direct analogue of the eigenvectors of a linear operator. Reducing subspaces are special invariant subspaces which are useful in the direct sum decomposition of an operator. They can also be used to classify an operator. The basic motivations for the study of invariant subspaces come from the interest in the structure of operators and from approximation theory to a wide variety of problems in physics (quantum theory), computer science (data mining), and chemistry (lattice theory of crystal analysis).

In particular, reducing subspaces find applications in wavelet expansion, multiresolution analysis (MRA) in image processing and automorphic graph theory.

If $A \in B(H)$ and $x \in H$, then $\bigvee_{n=0}^{\infty}\left\{A^{n} x\right\}$ is an invariant subspace of $A$. Therefore knowledge of $\operatorname{Lat}(A)$ gives information about the vectors which can be approximated by linear combinations of $\left\{A^{n} x\right\}$.

Knowledge of Hyperlat $(A)$ can give information about the structure of the commutant $\{A\}^{\prime}$ of $A$. Commutators of the form $A B-B A$ appear in a mathematical formulation of the Heisenberg's Uncertainty Principle.

\section{Conclusion}

In this paper, several concepts about subspace lattices have been introduced. It has been shown a unitary operator $T$, $\operatorname{Hyperlat}(T)=\operatorname{Re} d(T)$. It has also been proved that for a normal operator $T, \operatorname{Hyperlat}(T) \subseteq \operatorname{Hyperlat}\left(T^{*}\right)$, and that the following inclusions hold:

\section{Normal $\subset$ Reductive $\subset$ Reducible.}

\section{Acknowledgements}

The author wishes to pay tribute to the Eastern Africa University Mathematics Programme (EAUMP) for funding this research.

\section{References}

[1] H. Bercovici, C. Foias, and C. Pearcy, On the hyperinvariant subspace problem IV, Canadian J. Math. 60 (2008), 758-789.

[2] C. Foias, S. Hamid, C. Onica, and C. Pearcy, Hyperinvaraint subspaces III, J. Functional Anal. 222, No.1 (2005), 129-142. 
[3] M. F. Gamal, contractions: A Jordan model and lattices of invariant subspaces, St. Petersburg Math Journal 15 (2004), 773-793.

[4] L. V. Harkrishan, Elements of Hilbert spaces and operators, Springer, Singapore, 2017.

[5] D. Herrero, Quasisimilarity does not preserve the hyperlattice, Proc. Amer. Math. Soc. 65, No.1 (1977), 80-84.

[6] T. B. Hoover, Operator algebras with reducing invariant subspaces, Paci_c J. of Math. 44 (1973), 173-179.

[7] L. Kerchy, On the hyperinvariant subspace problem for asymptotically nonvanishing contractions, Operator Theory: Advances and Applications 127 (2001), 399-422.

[8] C. S. Kubrusly, An introduction to models and decompositions in operator theory, Birkhauser, Boston, 1997.

[9] C. S. Kubrusly, Elements of operator theory, Birkhauser, Basel, Boston, 2001.

[10] C. S. Kubrusly, Hilbert space operators:A problem solving approach, Birkhauser, Basel, Boston, 2003.
[11] C. S. Kubrusly, On similarity to normal operators, Mediterranean J. of Math. (2016), 2073-2085.

[12] W. E. Longstaff, A lattice-theoretic description of the lattice of hyperinvariant subspaces of a linear transformation, Can. J. Math. XXVIII, No. 5 (1976), 1062-1066.

[13] Valentine Matache, Operator equations and invariant subspaces, Le Matematiche XLIX-Fasc. I (1994), 143-147.

[14] A. Mello and C. S. Kubrusly, Quasiaffinity and invariant subspaces, Archiv der Mathematik 107 (2016), 173-184.

[15] R. Moore, Hyperinvariant subspaces of reductive operators, Proc. American Mathematical Society 63, No. 1 (1977), 9194.

[16] R. Moore, Reductive operators that commute with a compact operator, Michigan Math. J. 22 (1975), 229-233.

[17] M. Sababheh, A. Yousef and R. Khalil, On the invariant subspace problem, Bulletin of the Malaysian Math. Sci. Soc, April 2016, Vol. 39, Issue 2, 699-705. 